\title{
"Time-out from positive reinforcement" as the UCS in a CER paradigm with rats
}

HAROLD LEITENBERG, GREGORY J. BERTSCH, and RICHARD C. COUGHLIN, Jr., Department of Psychology, University of Vermont, Burlington, Vt. 05401

In the presence of a signal preceding time-out from positive reinforcement, and not in the presence of a control CS, rats' positively reinforced lever pressing behavior was suppressed, a result similar to what has often been obtained in the CER paradigm with a signal preceding electric shock. When time-out was withdrawn, response rate during the signal recovered; and when time-out was reinstated, response rate again declined. This finding with rats, however, is not in accord with previous findings with chimpanzees and pigeons whose ongoing positively reinforced behavior was accelerated rather than suppressed by a signal preceding time-out.

A signal preceding electric shock disrupts ongoing, positively reinforced behavior (Estes \& Skinner, 1941). This phenomenon is sometimes referred to as a conditioned emotional response (CER) or more descriptively as "conditioned suppression" (Hoffman \& Fleshler, 1961). A recently raised question (Leitenberg, 1965, 1966) is whether or not conditioned suppression is characteristic of signals preceding aversive stimuli other than electric shock.

For example, although time-out from positive reinforcement in large measure fulfills the negative reinforcement criteria of an aversive stimulus (Leitenberg, 1965), it has had an effect in the CER paradigm opposite to that of shock, i.e., conditioned acceleration. This accelerative effect on ongoing positively reinforced behavior of a pre-time-out signal has been demonstrated in separate studies with two chimpanzees (Ferster, 1958), three pigeons (Herrnstein, 1955), and seven pigeons (Leitenberg, 1966). In addition, with pigeons, conditioned acceleration has been reported not only with a stimulus signaling periods of non-reinforcement but also with a stimulus signaling periods of reduced frequency of positive reinforcement (Pliskoff, 1961, 1963).

If the reliability and generality of the conditioned acceleration finding could be confirmed, it might prove extremely fruitful in investigating the effects of drugs on different emotional behaviors. Since conditioned suppression has been considered a quantified reflection of "fear" when shock is employed, conditioned acceleration might be considered a quantified reflection of "frustration" when periods of nonreinforcement are substituted in place of shock in the CER paradigm. As a preliminary step toward performing such psychopharmacological studies, we attempted to reproduce the conditioned acceleration finding in another animal species, rats, using a discrimination procedure to control for pseudoconditioning.

\section{METHOD}

Eight male hooded rats maintained at $80 \%$ of free-feeding weight served as Ss. Each $S$ was magazine trained, shaped with food reinforcement to press the bar, and gradually trained to respond on a VI $7 \mathrm{~min}$ schedule. This schedule was selected because Herrnstein (1955) found acceleration during the CS preceding periods of nonreinforcement to be more likely when background reinforcement frequency and overall response rate were low.
Following shaping, Ss received 15 3-h sessions, in which each CS (a blinking of the house lights at a rate of 2 a sec and a click at a rate of $6 \mathrm{a} \mathrm{sec}$ ) was presented six times a session in a random sequence. During this phase, time-out from positive reinforcement (TO) did not follow its appropriate CS. CS duration was $30 \mathrm{sec}$, and there was a 15-min interval between the termination of one CS and the onset of the next. Reinforcement was available on the VI 7 min schedule during as well as between CS presentations.

Following this adaptation period, TO was introduced after its appropriate CS-the blinking light for four of the Ss and the click. for the others. The other CS was used as a control, signaling no change in the procedure. As in the adaptation phase each CS was presented six times per session. TO duration was $10 \mathrm{~min}$, and introduction of TOs increased the session length to $4 \mathrm{~h}$. During TO, bars were withdrawn from the experimental chambers so that the $S$ could make no response, nor receive any reinforcement.

Following 30 sessions with TO, 15 non-TO sessions, identical to the adaptation period, were introduced. These, in turn, were followed by an additional 30 sessions with TO being paired once again with one of the two CS.

\section{RESULTS}

Figure 1 compares the effects on ongoing behavior of the CS preceding time-out from positive reinforcement (TO) with the effects of the control CS. The results are expressed as a ratio: response frequency during each $\mathrm{CS}$ divided by response frequency during an equivalent period preceding each CS (pre-CS). A ratio below 1.00 indicates response suppression during the $C S$, and a ratio above 1.00 indicates acceleration. For half the Ss the CS signaling TO (pre-TO CS) was a clicking noise and the control CS was a tlashing light, while for the other half the stimuli were reversed. However, since the results were the same for the auditory and visual stimuli, the data for them were combined.

As can be seen in Fig. 1, the ratios for the CS preceding TO hovered around .8 when TO was present, and reverted back to the relatively high level of control $\mathrm{CS}$ ratios when TO was removed. On the other hand, although the ratios for the

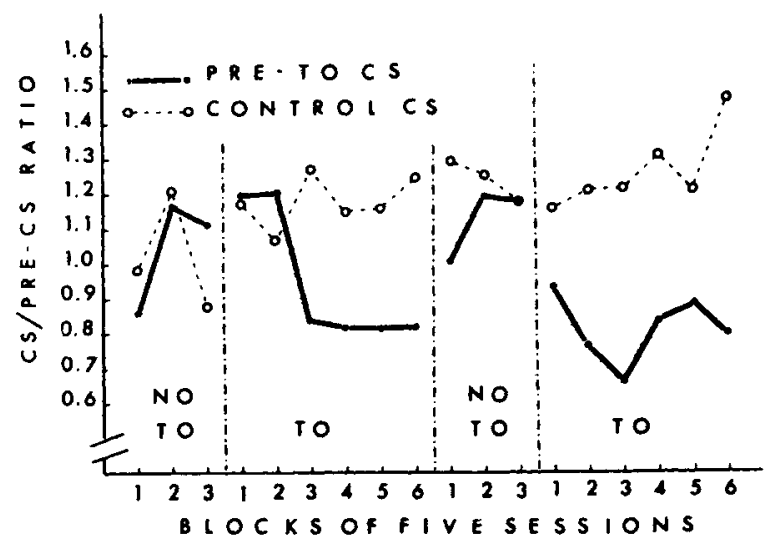

Fig. 1. Median CS/pre-CS ratios for CS preceding TO and control CS as a function of the presence and absence of TO. 


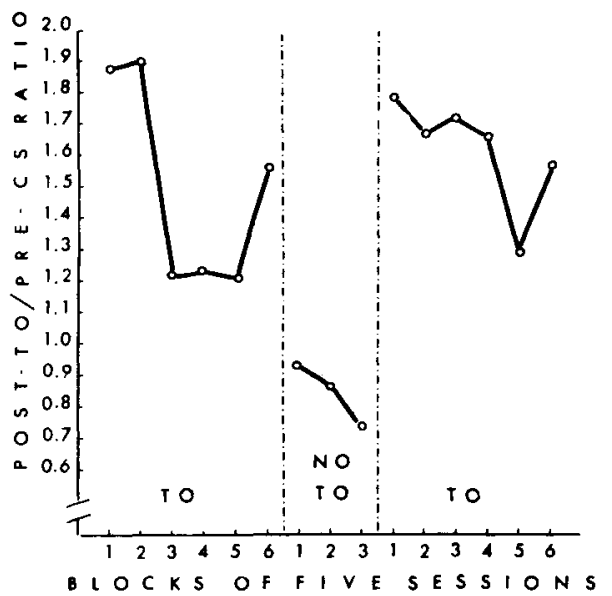

Fig. 2. Median post-TO/pre-CS ratios as a function of the presence and absence of TO.

control CS were consistently above 1.00 they did not change when TO was either introduced or removed.

In both phases (second and fourth) in which TO was present, responding in the presence of the pre-TO CS was suppressed relative to the control CS. The last 15 sessions of Phases 2 and 4 were combined for statistical analysis, and a two-tailed difference $t$ test (Edwards, 1960, p. 170) showed that the median ratio of the CS preceding TO was significantly lower than the median ratio of the control CS $(t=2.73$, $p<.05$ ). On the other hand, when TO was absent (Phases 1 and 3) a $t$ test showed no significant difference between the ratios associated with each CS.

Beginning with Phase 2, when TO was first introduced, a measure was taken of response frequency during a 30-sec period immediately subsequent to the conclusion of each TO. These data are plotted in Fig. 2 as a ratio to response frequency during corresponding $30-\mathrm{sec}$ pre-CS intervals. Following TO. Ss responded at a higher rate than during equivalent 30 -sec pre-CS periods $(t=4.51, p<.01)$. However, when TO was removed, response frequency during the period which formerly followed TO declined significantly $(t=3.65$, $p<.01)$. This finding of post-TO acceleration is perhaps analogous to "behavioral contrast" (Reynolds, 1961) or even to the "frustration effect" found in discrete trial situations (Amsel, 1958).

\section{DISCUSSION}

In the present study with rats, time-out from positive reinforcement (TO) had an effect similar to what has previously been reported for electric shock in the CER paradigm. As compared to the effects of a CS unpaired with TO, the signal preceding TO suppressed ongoing positively reinforced behavior. When TO was removed, this effect was reversed and when TO was reintroduced, this effect was reinstated. These findings are directly contradictory to previous studies (Herrnstein, 1955; Ferster, 1958, Experiment 1; Leitenberg, 1966) in which a signal preceding TO was found to accelerate rather than suppress ongoing behavior. The apparent major procedural difference between these earlier studies and the present one is that they used either pigeons or chimpanzees as Ss while this study used hooded rats.

In several other investigations, a number of procedural variables were concurrently changed to see if rats could be made "to behave like pigeons and chimpanzees," i.e., to exhibit conditioned acceleration in the CER paradigm when TO is used in place of electric shock. In one study, $(N=18)$ water deprivation and water reinforcement were used instead of food, tones were substituted for clicks, flashing side lights replaced flashing house lights, the VI schedule was 2 min instead of $7 \mathrm{~min}$, and the bar was left in the box instead of being withdrawn during TO. One experimental group received no reinforcement while the other received secondary reinforcement for each response made during $\mathrm{TO}$, and for control purposes, a pseudoconditioning group was used instead of differential conditioning within single Ss. The results were that the two experimental groups had suppression ratios of about .80 for 15 sessions; thereafter, they performed the same as the pseudoconditioning group hovering around the 1.00 level. In another study, TO duration was $15 \mathrm{~min}$ instead of 10 $\min , 22 \mathrm{~h}$ food deprivation was used instead of either water deprivation or $80 \%$ of normal body weight, and a multiple VI, FR schedule of food reinforcement was used, with VI values ranging from 2 to $5 \mathrm{~min}$, and $F R$ values ranging from 5 to 50 . The results were extremely variable for each $S(N=14)$ and the only conclusion that could be safely drawn was that the pre-TO CS was not exerting any powerful effect upon the instrumental behavior measured. Since none of these combined alterations resulted in conditioned acceleration, it is clear that the conditions necessary for obtaining this effect in rats have yet to be discovered. Although further research may yet succeed (especially if these variables are studied parametrically and one at a time), the failure so far to obtain qualitatively similar results in rats raises the possibility that the earlier data may be unreliable, i.e., not easily replicable in pigeons or chimpanzees either. This remains to be determined.

The results of the present study raise many more questions than they answer. Nevertheless, they serve a constructive function insofar as they correct a misleading picture in the literature of a secure finding: namely, that TO invariably produces an effect opposite to that of shock in the CER paradigm. This is clearly not the case.

\section{REFERENCES}

AMSEL, A. The role of frustrative nonreward in noncontinurous reward situations. Psychological Bulletin, 1958, 55, 102-119.

EDWARDS, A. L. Experimental design in psychological research. New York: Holt-Rinehart-Winston, 1960.

ESTES, W. K., \& SKINNER, B. F. Some quantitative properties of anxiety. Journal of Experimental Psychology, 1941, 29, 390-400.

FERSTER, C. B. Control of behavior in chimpanzees and pigeons by time-out from positive reinforcement. Psychological Monographs, 1958,72 , No. 8 (Whole No. 461).

HERRNSTEIN, R. J. Behavioral consequences of the removal of a discriminative stimulus associated with variable interval reinforcement. Unpublished doctoral thesis, Harvard Univ., 1955.

HOFFMAN, H. S., \& FLESHLER, M. Stimulus factors in aversive control: the generalization of conditioned suppression. Journal of the Experimental Analysis of Behavior, 1961, 4, 371-378.

LEITENBERG, $H$. Is time-out from positive reinforcement an aversive event? A review of the experimental evidence. Psychological Bulletin, $1965,64,428-441$.

LEITENBERG, $\mathrm{H}$. Conditioned acceleration and conditioned suppression in pigeons. Journal of the Experimental Analysis of Behavior, 1966, 9, 205-212.

PLISKOFF, S. S. Rate-change effects during a pre-schedule-change stimulus. Journal of the Experimental Analysis of Behavior, 1961, 4, 383-386.

PLISKOFF, S. S. Rate change effects with equal potential reinforcements during the "warning stimulus." Journal of the Experimental Analysis of Behavior, 1963, 6, 557-562.

REYNOLDS, G. S. Behavioral contrast. Journal of the Experimental Analysis of Behavior, 1961, 4, 57-71.

\section{NOTE}

1. This research was supported, in part, by N.I.M.H. Research Grant MH 12222-01 and by NSF Institutional Grant GU-1505 to the University of Vermont. While working on this project GJB was supported by an NSF pre-doctoral fellowship and RCG by an NDEA pre-doctoral fellowship. We thank James W. Stratton and John R. Bing (NSF undergraduate research participants) for contributing to the other investigations mentioned in this paper. Reprints may be obtained from the first author, Department of Psychology, University of Vermont, Burlington, Vermont, 05401 . 